\title{
Knowledge based Self Initializing FCM Algorithms for Fast Segmentation of Brain Tissues in Magnetic Resonance Images
}

\author{
T. Kalaiselvi \\ Assistant Professor \\ Department of Computer Science and Applications \\ Gandhigram Rural Institute - Deemed University \\ Dindigul District - 624302, Tamil Nadu, India
}

\author{
K. Somasundaram \\ Professor \\ Department of Computer Science and Applications \\ Gandhigram Rural Institute - Deemed University \\ Dindigul District - 624302, Tamil Nadu, India
}

\begin{abstract}
This paper presents two robust FCM algorithms to segment the brain tissues from MRI volume. The core regions of human brain are white matter (WM), gray matter (GM), cerebrospinal fluid (CSF) and others known as background (BCK). Usually, to classify the given data, random initial seeds are selected and then the FCM procedure is iterated until the centroids of cluster converge. The first proposed FCM, named as FCM-EXPERT, make use of the expert knowledge about the brain tissue properties to select the initial seed points. Experimental results on brain portions extracted from large MRI database show that this method is faster by 1.3 to 1.7 times than that of the traditional FCM in segmenting brain tissues. FCM-EXPERT has been again modified by making use of the correlation existing between brain regions in adjacent slices of MRI for centroid initialization and named as FCM-EXPERT-E. Experimental results of the second method show that this is faster by 2 to 4 times than that of traditional FCM.
\end{abstract}

\section{General Terms}

Image Segmentation, Clustering, Self Initialization.

\section{Keywords}

FCM clustering; brain tissues segmentation; centroid initialization; brain continuity; slice correlation; MRI scans.

\section{INTRODUCTION}

Segmentation plays an important role in biomedical image processing. It is often the starting point for other processes like analysis, visualization, quantization and modeling. This is a core technique to study about the anatomy and pathology of human organs. In brain diagnostic system, segmentation is essential to study any brain related disorders like seizures, stroke, multiple sclerosis, aneurisms, hemorrohages, brain tumors, brain cancers, brain atrophy, lesions, and sub structural changes. Three main regions of brain, white matter (WM), gray matter (GM) and cerebrospinal fluid (CSF) are the important subjects of study in brain diagnostic system. Manual segmentation of these three brain regions by an expert is time consuming, inconsistent and affected by operator bias as the volume of data involved in MRI studies is usually large. Hence an automatic segmentation of brain regions is essential for a physician to speed up their diagnostic process.

Segmentation techniques are broadly classified into two types: supervised and unsupervised [1]. Supervised methods require the user interaction and thus known as semi-automatic. Unsupervised techniques are completely automatic and segment the regions in feature space with a high density. Fuzzy C-Means (FCM) is an unsupervised clustering method. It automatically segments the region of interest (ROI) by classifying a tissue into several classes at the same time but with different degrees [2] [3] [4]. A study [5] revealed that FCM never misses a region and it is the best method for segmenting anisotropic nature of brain volumes that are affected by partial volume effect (PVE).

FCM algorithm employs fuzzy partitioning such that a data point can belong to all clusters with different membership grades between 0 and 1 . This method is frequently used in pattern recognition. The aim of FCM is to find cluster centers that minimize a dissimilarity (objective) function. By iteratively updating the cluster centers and the membership grade for each data point, FCM iteratively moves the cluster centers to the "right" location within a data set. But it does not ensure that it converges to an optimal solution in an optimal time, if the cluster centers (centroids) are initialized randomly. The performance of FCM depends on initial centroids of required clusters [6] [7]. Hence the selection of a centroid is important in FCM. For a robust approach there are two ways to select the centroids.

- Run the FCM several times each starting with different initial centroids and select the best initial values for the centroids

- Use application specific expert knowledge and select the initial centroids

The first approach becomes a supervised clustering technique and do not qualify to be a fully automatic method. Further it takes more time to identify the initial centroids. If the details about the data point density of the application are known prior then the second approach is preferable to generate the initial centroids.

Several modifications were proposed for FCM algorithm for medical image segmentation. Most of the existing works generally focused to frame a robust objective function to handle the noisy images [8] [9] [10], bias field estimation [11] [12] and sometimes both [13]. Nowadays advanced scanners are available that produce high resolution and non-artifact form of MRI images. So the modification for FCM algorithm is focused to fully automate its functionality based on the knowledge of the application area. This paper proposes such a modification to initialize the centroids of FCM method for brain tissue segmentation from MRI of head volume. 
This paper proposed two FCM methods that generate initial seeds by making use of the expert knowledge about the tissue properties appearing in the MRI of human head scans. FCM is a soft segmentation technique applicable for MRI brain tissues segmentation. The performance of FCM, to obtain an optimal solution depends on the selection of the initial positions of their centroids. In the existing FCM, the number of clusters is usually set by the users and their centroids are initialized randomly. This process generally takes time to reach the optimal solution. In order to accelerate the segmentation process, an application specific knowledge is used to initialize the centers of required clusters. To segment brain portion, the knowledge about the MRI intensity characteristics of brain regions is used to initialize the centroids. In the first method, FCM_EXPERT, the centroids are initialized using the intensity levels of the WM, GM, CSF and BCK. In the second method, FCM_EXPERT_E, the correlation existing between adjacent slices of an MRI volume is utilized. The final centroids obtained for the previous slice is used as initial centroid for the current slice. These methods are then applied on the brain portion extracted from MRI, and segmented the WM, GM and CSF.

The remaining part of this paper is organized as follows. In section 2, the existing FCM algorithm and the proposed methods used for brain tissue segmentation are given. In section 3, the materials used are given. The results and discussion are presented in section 4 . The conclusion is given in section 5 .

\section{METHOD}

\subsection{Fuzzy C-Means Method (FCM)}

FCM based clustering was originally introduced by Jim Bezdek in 1981 [3] [4]. The FCM algorithm attempts to partition a finite collection of elements $X=\left\{x_{1}, x_{2}, \ldots, x_{n}\right\}$ into a collection of c fuzzy clusters with centers $\mathrm{V}=v_{i}, \mathrm{i}=1,2, \ldots \mathrm{c}$ and a partition or membership matrix, $\mathrm{U}=u_{i j}, \mathrm{i}=1,2, \ldots \mathrm{n}, \mathrm{j}=1,2, \ldots \mathrm{c}$ where $u_{i j}$ is a numerical value in $[0,1]$ that tells the degree to which the element $x_{i}$ belongs to the $\mathrm{j}^{\text {th }}$ cluster.

The goal of FCM algorithm is to minimize the following objective function:

$$
J_{m}=\sum_{j=1}^{c} \sum_{i=1}^{n} u_{i j}^{m} d_{i j}
$$

where,

$$
d_{i j}=\left\|x_{i}-v_{j}\right\|
$$

$m \in[1, \infty]$ is a scalar termed as weighting exponent. $m$ controls the fuzziness of the resulting clusters and $d_{i j}$ is the Euclidian distance from object $x_{i}$ to the cluster center $v_{j}$. The centroid of the $j$ th cluster, $v_{j}$ is obtained as:

$$
v_{j}=\frac{\sum_{i=1}^{n} u_{i j}^{m} x_{i}}{\sum_{i=1}^{n} u_{i j}^{m}}
$$

where,

$$
u_{i j}=\frac{1}{\sum_{k=1}^{c}\left[\frac{d_{i j}}{d_{i k}}\right]^{\frac{2}{m-1}}}
$$

The following is the conventional FCM algorithm [3].

Step 1: Select the number of clusters c $(2 \leq c \leq n)$, exponential weight $\mathrm{m}$, initial membership matrix $\mathrm{U}$ and the termination/stopping criterion.

Step 2: Compute the cluster centers $v_{j}, j=1,2, \ldots, c$, according to equation (3)

Step 3: Compute Euclidian distance $d_{i j}, i=1,2, \ldots, n ; j=1,2, \ldots, c$ based on equation (2)

Step 4: Update the membership function $u_{i j}, i=1,2, \ldots, n ; j=1,2, \ldots, c$ using the equation (4)

Step 5: If not converged, go to step 2.

Step 6: Output $u_{i j}$ and $v_{j}$, the membership function and centroids of the data.

The FCM algorithm starts by setting values for the number of clusters (c), weighing factor $(\mathrm{m})$, initialization of $U$ and termination criterion as given in step 1 . The number of clusters is either supplied by the user during the process or automatically computed by the application. There is no fixed rule for choosing $\mathrm{m}$, however, in many applications $\mathrm{m}=2$ is a common choice. In case of crisp clustering ' $m$ ' may be chosen as 1.

The membership function matrix $u_{i j}$, contains the grade of membership of each object in each cluster. The values 0 and 1 indicate no membership and full membership respectively. Grades between 0 and 1 indicate that the object has partial membership in a cluster. Hence the summation of each row (total grades of an object) should be equal to unity. One more attribute of FCM is that it never leaves any region as blank [5]. These attributes are used to define the characteristics of fuzzy matrix $u_{i j}$ and are defined as follows [6]:

$u_{i j} \in[0,1] \quad \forall i=1,2, \ldots, n ; \forall j=1,2, \ldots, c$

$\sum_{j=1}^{c} u_{i j}=1 \quad \forall i=1,2, \ldots, n$ 
$0<\sum_{i=1}^{n} u_{i j}<n \quad \forall j=1,2, \ldots, c$

The FCM algorithm starts with the initialization of this membership function $u_{i j}$ as given in step 1 of FCM algorithm. In general, this is done by using random number generator and to satisfy the aforesaid three constraints of $u_{i j}$. Then the matrix $u_{i j}$ is used to generate the cluster centers (step 2 of FCM algorithm).

Several stopping criteria can be used for convergence. The most generalized convergence criteria can be stated as follows:

i. Set the number of iterations as maximum as possible, or

ii. The objective function $J_{m}$ defined in equation(1) cannot be minimized any further significantly, or

iii. The centroids no longer move in successive iterations.

Among these three criterions, the first two requires a user input in the form of number of iterations and value of error factor respectively. But the third one is a self generated one and does not require any external input. A comparative study [7] between these criteria showed that the third criterion yield results much faster than the other two. So the third is chosen as a stopping criterion for both the existing and proposed FCM methods.

\subsection{Proposed Method (FCM-EXPERT)}

As explained in the previous section, the performance of FCM algorithm depends on the selection of number of clusters $\mathbf{c}$ and their centroids $\mathbf{v}$. The major ROI of human brain image is $\mathrm{GM}, \mathrm{WM}, \mathrm{CSF}$ and the surrounding background (BCK). Hence for brain tissue segmentation $\mathbf{c}$ is fixed as 4 . The brain tissues are characterized by their intensity values in MRI scans. This expert knowledge is used to initialize the centroids. As the performance of the FCM depends on the initial position of the centroids, they can be initialized by selecting the intensity levels corresponding to the tissues instead of selecting them randomly. This will yield better results and quicken the process. This method is named as FCM-EXPERT.

\section{Imaging characteristics of MRI scans}

The images produced by MRI scans are usually gray images with intensity in the range $0-255$. The GM of the brain consists of the cortex that lines the external surface of the brain and the gray nuclei deep inside of the brain, including the thalami and basal ganglia. WM is comprised of the neuronal axons that interconnect different regions of the brain and serve as the interface between the brain and the rest of the body. The watery fluid, CSF acts as a cushion for physical shocks. The WM constitutes a connected region that is bordered by GM and CSF as shown in Fig.1. For the display purpose WM is shown in gray color, GM as white color and CSF as black color. In MRI of head scans, the picture of brain organ is usually surrounded by air particles, known as background, BCK in order to make a matrix representation. This BCK is another major ROI in MRI of head scans.

Jeny et al [14] analyzed the intensity characteristics of $\mathrm{T} 1$ MRI of head regions and generated a histogram of skull stripped image as shown in Fig.2. In the histogram there are three peaks, the first peak corresponds to CSF, the middle peak corresponds to gray matter and the last peak corresponds to white matter. The three peaks are used in their semiautomatic tool, BrainAssiat ${ }^{\mathrm{TM}}$ to separate the brain region. In this paper, the values of these peaks are used to initialize the centroids for the FCM method.

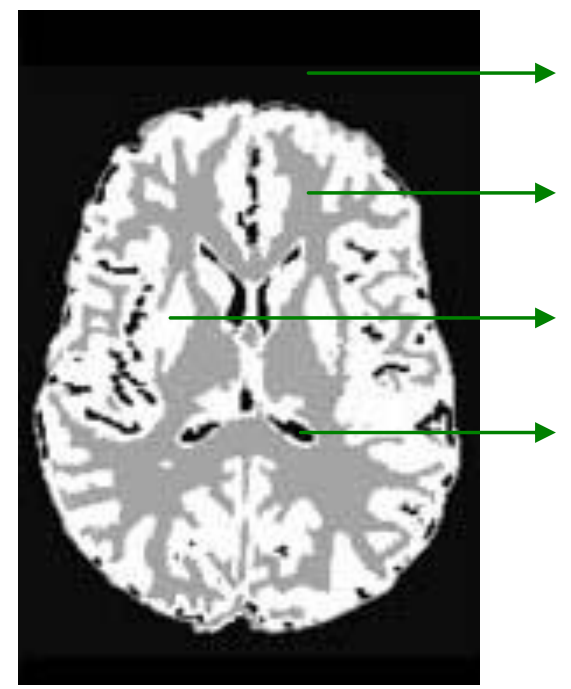

Background (BCK)

White Matter

(WM)

Gray matter

(GM)

Cerebrospinal fluid

(CSF)

Fig. 1. Segmentation results in MRI of axial head scan

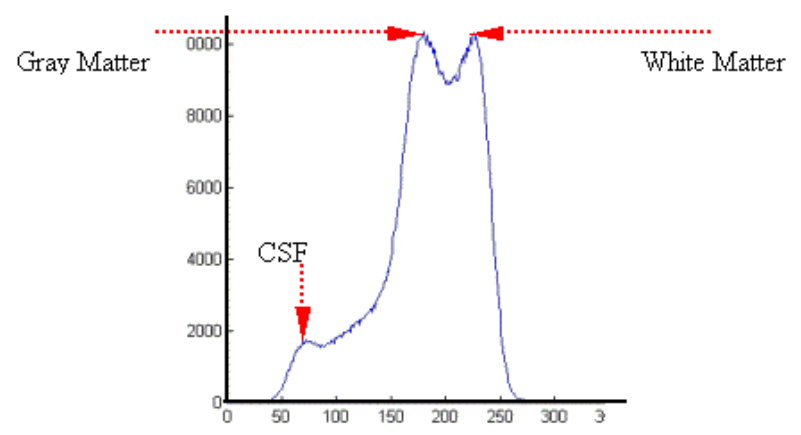

Fig. 2. Histogram of a skull stripped T1 weighted MRI

\section{Initialization of centroids}

In the intensity characteristics of MRI brain scans, the brain region lies in four major gray ranges, darkest, dark / dark gray, gray and bright representing BCK, CSF, GM, WM in T1 weighted images and BCK, WM, GM, CSF in T2 weighted images.

Hence the initial centroids for these four regions of gray scale image can be fixed as follow:

- The lowest intensity 0 is considered as cluster center for darkest region, always background (BCK)

- The highest intensity 255 is fixed as centroid for bright region

- In between, an equal interval is assumed based on the peaks of Fig. 2 and the values 85 and 170 are taken as centroids for dark gray and gray regions. 
Hence the intensity values $0,85,170$ and 255 are taken as initial centroids for the four regions to start the FCM algorithm in the proposed method, FCM-EXPERT.

The FCM-EXPERT algorithm is given below,

Step 1: Initialize the cluster centroids $v_{j}, j=1,2,3,4$ using the given values.

Step 2: Compute Euclidian distance $d_{i j}, i=1,2, \ldots, n ; j=1,2,3,4$ using equation (2)

Step 3: Compute the membership function $u_{i j}$, $i=1,2, \ldots, n ; j=1,2,3,4$ using equation (4)

Step 4: Update the cluster centers $v_{j}, j=1,2,3,4$, using equation (3)

Step 5: If none of the centroids $\left(v_{j}, j=1,2,3,4\right)$ changes in step 4 , stop; otherwise go to step 2

Step 6: Output $u_{i j}$ and $v_{j}$, the membership function and centroids of the data.

\subsection{Extension of the proposed Method (FCM-EXPERT-E)}

In MRI of head scan, there is a continuity of the brain portion between two adjacent slices. Therefore there should be high correlation between the two adjacent brain areas. Brummer et al., [15] have suggested that this continuity property of brain in a volume could be exploited in neighboring slices to select the ROI. The brain extraction algorithms (BEA) [15] [16] [17] used this concept to extract the brain volume from the MRI volume. This property is used to quicken the proposed FCMEXPERT method and named it as FCM-EXPERT-E. The flowchart for FCM-EXPERT-E is shown in Fig.3.

The algorithm for FCM-EXPERT-E is given below.

Step 1: Input the MRI of head scans (volume).

Step 2: Extract the brain volume from MRI volume using BEA.

Step 3: Find the middle slice (M) from the MRI brain volume.

Step 4: Divid the brain volume into two sub volumes: lower slices (LS) lying below the $\mathrm{M}$ and upper slices (US) lying above $\mathrm{M}$.

Step 5: The LS range is M-1 to bottom-end (BE) of volume and US range is $\mathrm{M}+1$ to top-end (TE) of volume

Step 6: Apply the FCM-EXPERT on the middle slice M by taking initial centroids as $0,85,170,255$ and compute the final centroids.

Step 7: Move one slice up in US volume (one slice down in LS volume) by taking the final centroids of the previous slice as initial centroids and apply FCMEXPERT.

Step 8: Repeat step 7 until TE (BE) is reached.

\section{MATERIALS}

Twenty datasets of $\mathrm{T} 1$ and $\mathrm{T} 2$ types obtained from different sources are used for the experiments. Ten T1 coronal volumes of normal subjects were obtained from the 'Internet Brain Segmentation Repository' (IBSR) developed by Centre for Morphometric Analysis (CMA) at Massachusetts General Hospital [19]. Each IBSR dataset has approximately 60 slices per volume with slice thickness = contiguous $3.1 \mathrm{~mm}$ and slice matrix $=256 \times 256$. Another ten $\mathrm{T} 2$ axial datasets consisting of normal and abnormal volumes with brain tumor were taken from the website 'The Whole Brain Atlas' (WBA) maintained by Department of Radiology and Neurology at Brigham and Women's Hospital, Harvard Medical School [20], and from KGS Advanced MR and CT Scans, India. WBA volume consists approximately 25 slices with slice size $256 \times 256$ and slice thickness $\approx 5 \mathrm{~mm}$. KGS has approximately 20 slices per volume with slice matrix $=448 \times 512$ and slice thickness $\approx 7 \mathrm{~mm}$ along with inter slice gap. KGS scans are much affected by partial volume effect (PVE) artifact.

\section{RESULTS/DISCUSSION}

The experiments are carried out using the conventional FCM algorithm and the two proposed algorithms FCM-EXPERT and FCM-EXPERT-E on the images chosen from the material pool.

Initially a qualitative performance evaluation of these methods on original MR scan was done. The segmentation results obtained are shown in Fig.4. The first row shows the segmentation results obtained for original MRI of head scan and the second row for skull stripped brain portion of the same slice. For the original image given in row 1 of Fig.4, some of the surrounding non-brain regions like fat, skin, bone and neck portions are misclassified as the required ROIs. But when these methods were applied on brain portion extracted from the original image, the FCM algorithm gave more accurate classifications as given in row 2 of Fig.4. This type of testing was done on several volumes and found that the segmentation on extracted brain produced accurate results. Hence, for further validation, the methods were applied on the brain portion extracted from the MR scans using the brain extraction algorithms [15] [16].

The conventional FCM algorithm and proposed procedures FCM-EXPERT and FCM-EXPERT-E were applied on the datasets of different types of images and orientations taken from the material pool. In each orientation, only the slices with major brain regions were taken for experiments leaving the initial and trailing slices appearing at both ends of the volume. Middle 40 slices out of 60 for IBSR T1 coronal volumes and 10 slices out of 20 for WBA and KGS T2 axial volumes were taken. The conventional FCM algorithm with random initialization was applied on the brain portion in order to find the optimal solution in terms of number of iterations, cluster centers and time taken for processing the images. The values computed are given in Table 1 . Then the algorithm FCM-EXPERT with the initial centroids as $0,85,170,255$ was applied on the same brain portion. The computed values are given in Table 1. From Table 1, it was noticed that the average number of iterations for the traditional FCM is 19, 21 and 24 for IBSR, WBA and KGS datasets respectively. But the proposed FCM-EXPERT reduced these values to 13, 14 and 18 respectively. Hence the processing times were also reduced considerably. This shows that the proposed FCMEXPERT is 1.4, 1.72 and 1.32 times faster than the existing FCM for the normal (IBSR), abnormal (WBA) and PVE (KGS) datasets. 


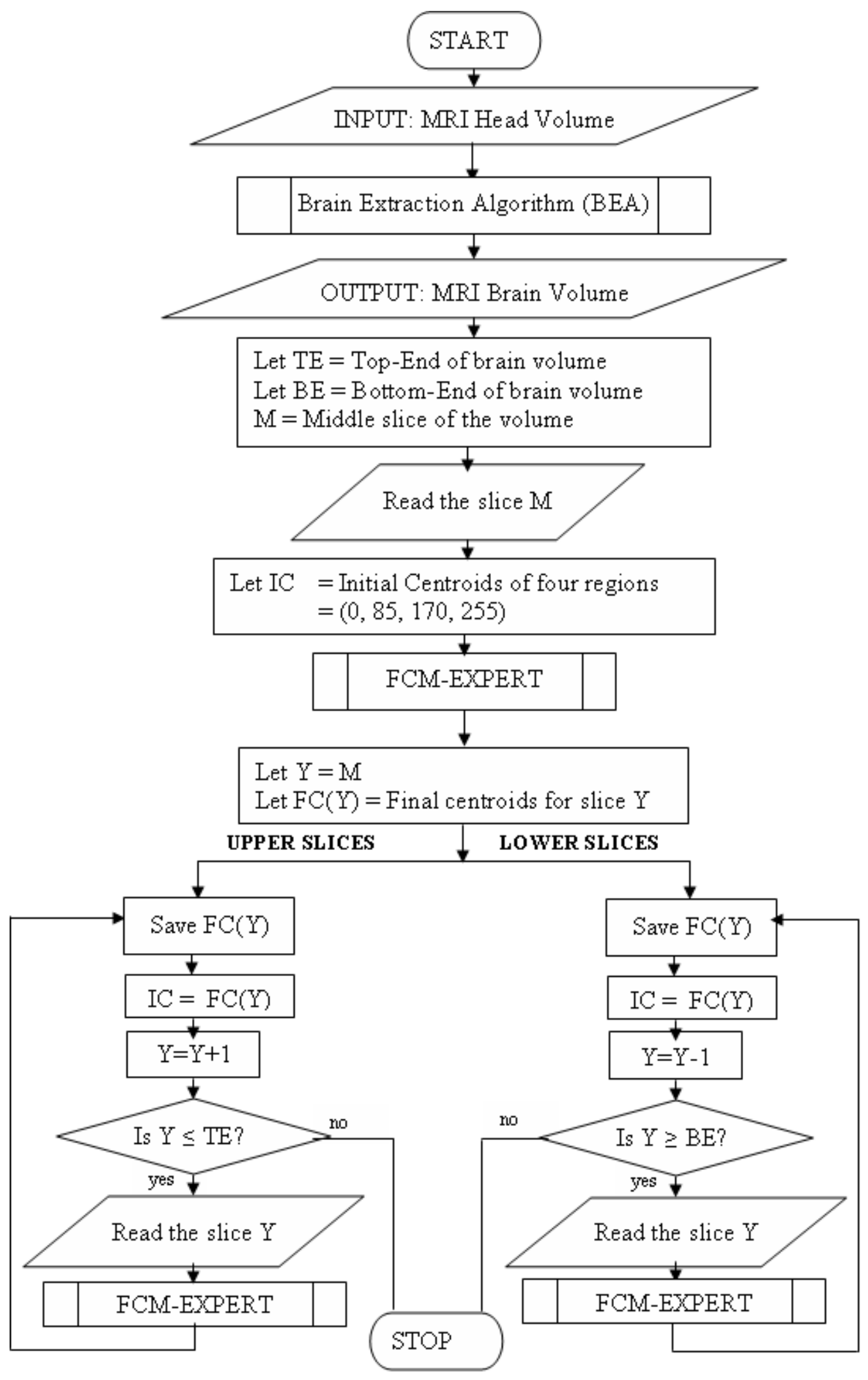

Fig. 3. Flowchart of FCM-EXPERT-E 

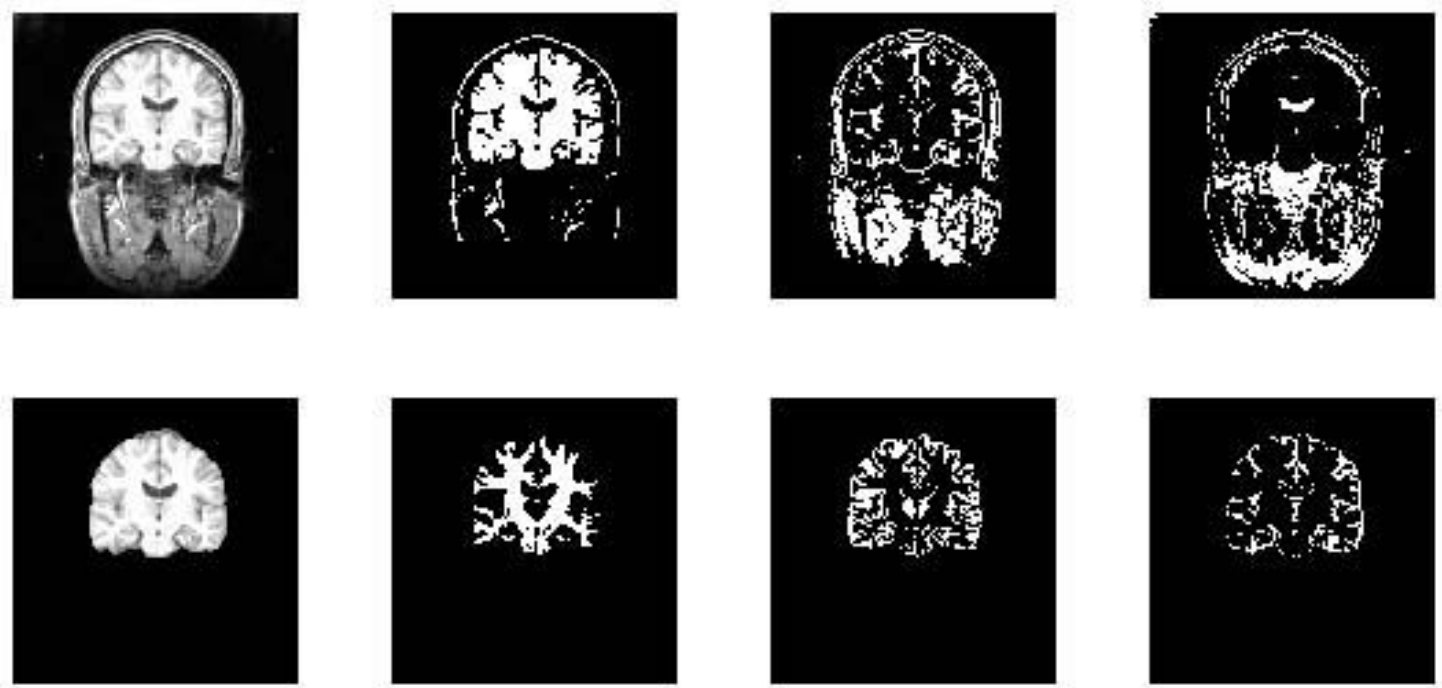

Fig. 4. Segmentation results on raw and extracted brain image. Row 1 shows the segmentation of raw MRI scans and row2 shows the segmentation of the brain portion extracted from the original. Column 1 shows the original head scan and the extracted brain in row 1 and row2. The three ROIs, WM, GM and CSF of head and brain obtained by FCM are in column 2-4 respectively.

Table 1. The average number of iterations, cluster centers and segmentation time of MRI volumes

\begin{tabular}{|c|c|c|c|c|c|c|c|c|c|c|c|c|c|c|c|c|c|c|c|}
\hline \multirow{4}{*}{$\begin{array}{l}\text { Data } \\
\text { Source }\end{array}$} & \multirow{4}{*}{$\begin{array}{c}\text { Data } \\
\text { set }\end{array}$} & \multicolumn{18}{|c|}{ FCM Method } \\
\hline & & \multicolumn{6}{|c|}{ FCM Conventional } & \multicolumn{6}{|c|}{ FCM-EXPERT } & \multicolumn{6}{|c|}{ FCM-EXPERT-E } \\
\hline & & \multirow{2}{*}{$\begin{array}{l}\text { No. } \\
\text { iter }\end{array}$} & \multicolumn{4}{|c|}{ Final Centroids } & \multirow{2}{*}{$\begin{array}{l}\text { Proc. } \\
\text { Time }\end{array}$} & \multirow{2}{*}{$\begin{array}{l}\text { No. } \\
\text { iter }\end{array}$} & \multicolumn{4}{|c|}{ Final Centroids } & \multirow{2}{*}{$\begin{array}{l}\text { Proc. } \\
\text { Time }\end{array}$} & \multirow{2}{*}{$\begin{array}{l}\text { No. } \\
\text { iter }\end{array}$} & \multicolumn{4}{|c|}{ Final Centroids } & \multirow{2}{*}{$\begin{array}{l}\text { Proc. } \\
\text { Time }\end{array}$} \\
\hline & & & C1 & $\mathbf{C 2}$ & $\mathrm{C3}$ & C4 & & & C1 & $\mathbf{C 2}$ & C3 & C4 & & & C1 & C2 & $\mathbf{C 3}$ & C4 & \\
\hline \multirow{11}{*}{ IBSR } & vol 1 & 19 & 0 & 143 & 202 & 249 & 4.74 & 14 & 0 & 143 & 202 & 249 & 3.33 & 4 & 0 & 144 & 203 & 249 & 0.778 \\
\hline & vol 2 & 19 & 0 & 126 & 172 & 217 & 3.29 & 10 & 0 & 126 & 172 & 217 & 2.11 & 8 & 0 & 127 & 173 & 217 & 1.447 \\
\hline & vol 3 & 19 & 0 & 136 & 193 & 243 & 3.49 & 13 & 0 & 135 & 192 & 243 & 2.37 & 5 & 0 & 137 & 194 & 244 & 0.896 \\
\hline & vol 4 & 19 & 0 & 140 & 194 & 248 & 3.70 & 13 & 0 & 138 & 193 & 248 & 2.40 & 6 & 0 & 140 & 194 & 248 & 1.034 \\
\hline & vol 5 & 18 & 0 & 144 & 207 & 248 & 3.15 & 14 & 0 & 143 & 206 & 248 & 2.65 & 4 & 0 & 144 & 207 & 248 & 0.78 \\
\hline & vol 6 & 19 & 0 & 138 & 196 & 246 & 3.34 & 13 & 0 & 137 & 195 & 246 & 2.24 & 4 & 0 & 139 & 196 & 247 & 0.76 \\
\hline & vol 7 & 18 & 0 & 144 & 208 & 250 & 3.35 & 14 & 0 & 143 & 208 & 249 & 2.55 & 5 & 0 & 144 & 209 & 250 & 0.793 \\
\hline & vol 8 & 18 & 0 & 140 & 203 & 249 & 3.26 & 13 & 0 & 140 & 203 & 249 & 2.47 & 4 & 0 & 141 & 204 & 249 & 0.75 \\
\hline & vol 9 & 18 & 0 & 134 & 200 & 247 & 3.11 & 13 & 0 & 134 & 199 & 247 & 2.23 & 4 & 0 & 135 & 200 & 248 & 0.778 \\
\hline & vol 10 & 18 & 0 & 136 & 204 & 249 & 3.07 & 13 & 0 & 135 & 203 & 249 & 2.26 & 4 & 0 & 136 & 204 & 250 & 0.757 \\
\hline & Avg & 19 & $\mathbf{0}$ & 138 & 198 & 245 & 3.45 & 13 & $\mathbf{0}$ & 137 & 197 & 245 & 2.46 & 5 & $\mathbf{0}$ & 139 & 198 & 245 & 0.877 \\
\hline \multirow{6}{*}{ WBA } & vol 11 & 20 & 0 & 89 & 128 & 195 & 4.85 & 13 & 0 & 90 & 130 & 198 & 2.99 & 9 & 0 & 90 & 130 & 197 & 2.145 \\
\hline & vol 12 & 21 & 0 & 75 & 114 & 208 & 5.10 & 16 & 0 & 76 & 116 & 210 & 3.10 & 7 & 0 & 76 & 115 & 210 & 1.172 \\
\hline & vol 13 & 23 & 0 & 84 & 124 & 201 & 5.00 & 15 & 0 & 84 & 127 & 204 & 3.02 & 9 & 0 & 84 & 125 & 203 & 1.527 \\
\hline & vol1 4 & 21 & 0 & 112 & 147 & 208 & 4.62 & 9 & 0 & 113 & 150 & 211 & 1.68 & 6 & 0 & 112 & 149 & 210 & 1.07 \\
\hline & vol 15 & 21 & 0 & 79 & 121 & 187 & 5.64 & 15 & 0 & 80 & 124 & 188 & 3.84 & 6 & 0 & 80 & 122 & 187 & 1.492 \\
\hline & Avg & 21 & 0 & 88 & 127 & 200 & 5.04 & 14 & 0 & 89 & 129 & 202 & 2.93 & 7 & 0 & 88 & 128 & 201 & 1.481 \\
\hline \multirow{6}{*}{ KGS } & vol 16 & 23 & 0 & 69 & 97 & 166 & 15.10 & 17 & 0 & 70 & 100 & 169 & 11.68 & 10 & 0 & 70 & 98 & 167 & 5.752 \\
\hline & vol 17 & 21 & 0 & 69 & 97 & 153 & 12.89 & 19 & 0 & 70 & 99 & 157 & 11.44 & 9 & 0 & 70 & 98 & 156 & 5.225 \\
\hline & vol 18 & 26 & 0 & 73 & 103 & 162 & 15.33 & 17 & 0 & 74 & 104 & 165 & 10.45 & 11 & 0 & 74 & 100 & 159 & 6.528 \\
\hline & vol 19 & 23 & 0 & 71 & 100 & 163 & 14.26 & 17 & 0 & 72 & 103 & 167 & 10.86 & 10 & 0 & 74 & 104 & 164 & 5.661 \\
\hline & vol 20 & 25 & 0 & 73 & 98 & 156 & 16.59 & 18 & 0 & 74 & 101 & 160 & 11.65 & 9 & 0 & 72 & 103 & 166 & 5.378 \\
\hline & Avg & 24 & 0 & 71 & 99 & 160 & 14.83 & 18 & 0 & 72 & 101 & 164 & 11.22 & 10 & $\mathbf{0}$ & 72 & 101 & 162 & 5.709 \\
\hline
\end{tabular}


Table 2. Performance of FCM-EXPERT-E for vol 5 of IBSR

\begin{tabular}{|c|c|c|c|c|c|c|c|}
\hline Slices & Slice No & $\begin{array}{c}\text { No. of } \\
\text { Iter. }\end{array}$ & C1 & $\mathrm{C2}$ & C3 & C4 & $\begin{array}{c}\text { Pro. Time } \\
\text { (Sec) }\end{array}$ \\
\hline $\begin{array}{c}\text { Middle Slice } \\
\text { (M) }\end{array}$ & 30 & 15 & $\mathbf{0}$ & 149 & 210 & 250 & 2.562 \\
\hline \multirow{19}{*}{$\begin{array}{c}\text { Lower Slices } \\
\text { (LU) }\end{array}$} & 29 & 3 & $\mathbf{0}$ & 149 & 208 & 251 & 0.531 \\
\hline & 28 & 4 & 0 & 149 & 209 & 250 & 0.703 \\
\hline & 27 & 6 & 0 & 143 & 209 & 249 & 1.031 \\
\hline & 26 & 7 & 0 & 138 & 206 & 249 & 1.204 \\
\hline & 25 & 4 & 0 & 139 & 205 & 248 & 0.703 \\
\hline & 24 & 4 & 0 & 138 & 205 & 248 & 0.687 \\
\hline & 23 & 4 & 0 & 140 & 203 & 248 & 0.687 \\
\hline & 22 & 4 & 0 & 139 & 204 & 249 & 0.703 \\
\hline & 21 & 4 & 0 & 137 & 204 & 248 & 0.688 \\
\hline & 20 & 3 & 0 & 137 & 204 & 247 & 0.515 \\
\hline & 19 & 5 & 0 & 135 & 203 & 246 & 0.875 \\
\hline & 18 & 3 & 0 & 136 & 201 & 245 & 0.531 \\
\hline & 17 & 4 & 0 & 140 & 201 & 243 & 0.687 \\
\hline & 16 & 4 & 0 & 141 & 200 & 243 & 0.703 \\
\hline & 15 & 4 & 0 & 143 & 201 & 242 & 0.703 \\
\hline & 14 & 6 & 0 & 146 & 202 & 242 & 1.032 \\
\hline & 13 & 4 & 0 & 147 & 204 & 244 & 0.688 \\
\hline & 12 & 4 & 0 & 146 & 206 & 245 & 0.703 \\
\hline & 11 & 3 & 0 & 144 & 205 & 246 & 0.531 \\
\hline \multirow{20}{*}{$\begin{array}{l}\text { Upper Slices } \\
\text { (US) }\end{array}$} & 31 & 5 & 0 & 145 & 208 & 250 & 0.86 \\
\hline & 32 & 4 & 0 & 149 & 209 & 250 & 0.703 \\
\hline & 33 & 5 & 0 & 151 & 210 & 250 & 0.86 \\
\hline & 34 & 3 & 0 & 153 & 209 & 250 & 0.515 \\
\hline & 35 & 7 & 0 & 146 & 210 & 250 & 1.203 \\
\hline & 36 & 5 & 0 & 143 & 209 & 249 & 0.875 \\
\hline & 37 & 4 & 0 & 143 & 208 & 248 & 0.688 \\
\hline & 38 & 3 & 0 & 143 & 206 & 248 & 0.531 \\
\hline & 39 & 4 & 0 & 146 & 205 & 248 & 0.687 \\
\hline & 40 & 4 & 0 & 147 & 206 & 249 & 0.688 \\
\hline & 41 & 3 & 0 & 147 & 206 & 249 & 0.531 \\
\hline & 42 & 6 & 0 & 147 & 210 & 251 & 1.156 \\
\hline & 43 & 4 & 0 & 151 & 212 & 251 & 0.687 \\
\hline & 44 & 4 & 0 & 148 & 211 & 251 & 0.688 \\
\hline & 45 & 3 & 0 & 151 & 211 & 251 & 0.532 \\
\hline & 46 & 4 & 0 & 151 & 212 & 251 & 0.703 \\
\hline & 47 & 5 & 0 & 145 & 213 & 251 & 0.875 \\
\hline & 48 & 4 & 0 & 141 & 213 & 251 & 0.703 \\
\hline & 49 & 3 & 0 & 140 & 213 & 251 & 0.532 \\
\hline & 50 & 4 & 0 & 138 & 213 & 250 & 0.703 \\
\hline Average & & 4 & 0 & 144 & 207 & 248 & 0.78 \\
\hline
\end{tabular}

Finally, the proposed FCM_EXPERT-E was applied on the selected MRI volumes. Table 2 shows the relative change of centroids and iterations for lower slices (LS), upper slices (US) and the middle slice (M) for vol 5 of IBSR. It is observed that only the slice $M$ requires 15 iterations to reach the convergence. The final centroid produced for slice $M$ is used to initialize the adjacent slices 29 and 31, lying above and below it. In this way the resultant centroids of each slice is used to initialize their adjacent slice and executed the FCMEXPERT-E algorithm.

The average values computed using FCM-EXPERT-E for the selected volumes are given in Table 1. From Table 1, it is noticeable that the average number of iterations for conventional FCM is 19 whereas it is reduced to 13 by proposed FCM_EXPERT and to 5 by FCM_EXPERT-E. The average segmentation time are 0.877 seconds/slice for IBSR, 
1.481 seconds/slice for WBA and 5.709 seconds/slice for KGS. This method is 3.93 times faster than conventional FCM and 2.8 times faster than FCM-EXPERT of IBSR volumes. For WBA, it is observed that the proposed method FCM-EXPERT-E is 3.4 times faster than conventional FCM and 1.98 faster than FCM-EXPERT. For KGS datasets, it is found the same trend and the proposed method FCMEXPERT-E is 2.6 and 1.97 times faster than conventional FCM and FCM-EXPERT. This shows that the proposed FCM-EXPERT-E based segmentation is 3 to 4 times faster for normal volumes, 2 to 3 times faster for abnormal volumes and 2 times faster for PVE volumes than the conventional FCM and FCM-EXPERT method.

The experiments were performed in a $1.73 \mathrm{GHz}$ Intel Pentium dual-core processor, Windows XP with 1GB RAM, using Matlab 6.5.

\section{CONCLUSION}

The performance of Fuzzy-C-Means (FCM) clustering algorithm depends on the initialization of the seed points. This paper proposed two novel seed initialization methods for the conventional FCM algorithm. The initialization is done based on the domain specific expert knowledge of the MRI of human head scans. In the first method, the seed initialization is done using the WM, GM and CSF properties in MRI. Application of this method on brain portion extracted from publically available T1 and T2 weighted MRI of human head scans show that the first proposed method FCM_EXPERT is faster by 1.32 to 1.72 times than that of the traditional FCM in segmenting WM, GM and CSF. In the second seed initialization method, FCM-EXPERT-E, the correlation existing between the adjacent slices of an MRI volume is exploited. This method is faster by 2 to 4 times than the conventional method in segmenting WM, GM and CSF. The experiments show that a domain specific knowledge can be effectively used to initialize the centroids of FCM method.

\section{ACKNOWLEDGMENTS}

The authors wish to thank Dr. K.G. Srinivasan MD., RD., Consultant Radiologist, KGS Advanced MR \& CT Scan, Madurai, Tamilnadu, India for providing the MR Head scans.

\section{REFERENCES}

[1] Jain, A. K., Murty, M. N. and Flynn, P. J. 1999. Data Clustering - A Review, ACM Computing Survey, 31(3), 265-322.

[2] Dunn, J. C. 1973. A Fuzzy Relative of the ISODATA Process and Its Use in Detecting Compact WellSeparated Clusters, Journal of Cybernetics, 3, 32-57.

[3] Bezdek, J. C. 1981. Pattern Recognition with Fuzzy Objective Function Algorithms, Plenum Press, New York.

[4] Pal, N.R. and Bezdek, J.C. 1995. On Cluster Validity for the Fuzzy C-Means Model, IEEE Transactions on Fuzzy Systems, 3(3), 370-379.

[5] Somasundaram, K. and Kalaiselvi, T. 2009. A Comparative Study of Segmentation Techniques Used for MR Brain Images, International Conference on Image Processing, Computer Vision, and Pattern Recognition, Las Vegas, Nevada, USA, (July 13-16), 597-603.

[6] Izakian, H. and Abraham, A. 2011. Fuzzy C-means and Fuzzy Swarm for Fuzzy Clustering Problem, Expert Systems with Applications, 38, 1835-1838.
[7] Kalaiselvi, T. and Somasundaram, K. 2010. Fuzzy CMeans Technique with Histogram Based Centroid Initialization for Brain Tissue Segmentation in MRI of Head Scans, IEEE sponsored 2011 International Symposium on Humanities, Science \& Engineering Research - SHUSER2011, Kuala Lumpur, Malaysia, (June 5-7), 155-160

[8] Kannan, S.R., Ramathilagam, S., Devi, R. and Sathya, A. 2011. Robust kernel FCM in segmentation of breast medical images, Expert Systems with Application, 38, $4382-4389$

[9] Wang, J., Kong, J., Lu, Y., Qi, M. and Zhang, B. 2008. A modified FCM algorithm for MRI brain image segmentation using both local and non-local spatial constraints, Computerized Medical Imaging and Graphics, 32(8), 685-698.

[10] Chuang, K.S., Tzeng, H.L., Chen, S., Wu, J. and Chen, T.J. 2006. Fuzzy c-means clustering with spatial information for image segmentation, Computerized Medical Imaging and Graphics, 30, 9-15.

[11] Ji, Z.X., Sun, Q.S. and Xia, D.S. 2011. A modified possibilistic fuzzy c-means clustering algorithm for bias field estimation and segmentation of brain MR image, Computerized Medical Imaging and Graphics, 35(5), 383-397.

[12] Ahmed, M.N., Yamany, S.M., Mohamed, N., Farag, A.A. and Moriarty, T. 2002. A Modified Fuzzy C-Means Algorithm for Bias Field Estimation and Segmentation of MRI Data, IEEE Transactions on Medical Imaging, 21(3), 193-199.

[13] Moussaoui, A., Benmahammed, K., Ferahta, N. and Chen, V. 2005. A New MR Brain Image Segmentation Using an Optimal Semi-supervised Fuzzy C-Means and pdf Estimation, Electronic Letters on Computer Vision and Image Analysis, 5(4), 1-11.

[14] Jeny, R., Kannan, K., Thomas, F., Kesavadas, C. and Chandrasekhar, P. S. 2008. MR Brain volume Analysis Using BrainAssist, International Conference on Systematic, Cybernetics and Informatics - ICSCI2008, India, (January 2008).

[15] Somasundaram, K. and Kalaiselvi, T. 2010. Brain Extraction Method for T1 Magnetic Resonance Scans, IEEE International Conference on Signal Processing and Communication, Indian Institute of Sciences (IISc), Bangalore, India, (July 18-21), 1-5.

[16] Somasundaram, K. and Kalaiselvi, T. 2010. Fully Automatic Brain Extraction Algorithm for Axial T2Weighted Magnetic Resonance Images, Computers in Biology and Medicine, 40(10), 811-822.

[17] Somasundaram, K. and Kalaiselvi, T. 2011. Automatic Brain Extraction Methods for T1 Magnetic Resonance Images using Region Labeling and Morphological Operations, Computers in Biology and Medicine, 41(8) 716-725.

[18] Brummer, M.E., Mersereau, R.M., Eisner, R.L. and Lewine, R.J. 1993. Automatic Detection of Brain Contours in MRI Datasets, IEEE Transactions on Medical Imaging, 12(2), 153-166.

[19] http://www.cma.mgh.harvard.edu/ibsr/index.html

[20] http://www.med.harvard.edu/AANLIB/home.html 